\title{
ERRATUM
}

Simon M. Gunn · Grant E. van der Ploeg

Robert T. Withers · Christopher J. Gore

Neville Owen · Adrian E. Bauman · John Cormack

\section{Measurement and prediction of energy expenditure in males during household and garden tasks}

Published online: 31 March 2004

(C) Springer-Verlag 2004

\section{Eur J Appl Physiol (2004) 91:61-70}

Unfortunately, this paper contains the following error:

The $\mathrm{CSA}_{\text {arm }}$ coefficient for lawn mowing in Table 4 (p. 66) should be $4.5 \times 10^{-4}$.

The online version of the original article can be found at http:// dx.doi.org/10.1007/s00421-003-0932-1

S. M. Gunn · G. E. van der Ploeg · R. T. Withers $(\bowtie)$

Exercise Physiology Laboratory, School of Education,

Flinders University, GPO Box 2100, Adelaide,

South Australia, Australia 5001

E-mail: bob.withers@flinders.edu.au

Tel.: + 61-8-82012256

Fax: + 61-8-82013407

C. J. Gore

Australian Institute of Sport, PO Box 21,

Henley Beach, South Australia, Australia 5022

N. Owen

School of Population Health, The University of Queensland,

Herston, Queensland, Australia 4006

A. E. Bauman

University of New South Wales and Epidemiology Unit, South-West Sydney Area Health Service, Locked Bag 7017. Liverpool BC 1871, New South Wales, Australia 2170

J. Cormack

Division of Medical Imaging, Flinders Medical Centre,

Bedford Park, South Australia, Australia 5042 